\section{The Reliability and Validity of a Handheld Relative Afferent Pupillary Tester in Diagnosing Optic Neuropathy}

\author{
Timothy T You ${ }^{1 *}$, Crystal X Huang ${ }^{2}$, Sanford Chen ${ }^{1}$, John \\ Maggiano', Rajiv Rathod ${ }^{1}$, Eugene Chang', Marinel Casiano ${ }^{2}$ \\ and Babak Kamkar ${ }^{3}$
}

'Orange County Retina Group, Santa Ana, California, USA

${ }^{2}$ Orange County Retina Research, Santa Ana, California, USA

${ }^{3}$ Newport Harbor Optometry, Costa Mesa, California, USA

\begin{abstract}
Context: With the conventional methodology of the swinging flashlight test, examiners focus on just one pupil at a time to detect cases of optic neuropathy. In contrast, the APD Tester ${ }^{\mathrm{TM}}$ allows for simultaneous comparison of relative pupillary responses.

Aims: To present the first case series on use of the APD Tester ${ }^{\mathrm{TM}}$, a new device that improves the ability to detect relative afferent pupillary defects.

Settings and Design: Observational case series and instrument validation.

Methods and Material: The examiner's left eye views the subject's right pupil and the examiner's right eye views the subject's left pupil, simultaneously in a superimposed image. Direct and consensual pupillary responses are observed, as bright lights alternately illuminate the subject's pupils.

Results: We present data on the use of the APD Tester ${ }^{\mathrm{TM}}$ for 10 known normal subjects and 10 subjects with optic neuropathy in one eye.

Conclusion: Using the APD Tester ${ }^{\mathrm{TM}}$, examiners may detect relative afferent pupillary defects with greater ease and clarity than afforded by standard testing methods, in which examiners view each eye individually.

Keywords: APD tester; Consensual pupillary response; Direct pupillary response; Optic neuropathy; Relative afferent pupillary defect Key Message: The APD Tester ${ }^{\mathrm{TM}}$ is a new device that facilitates accurate detection and quantification of relative afferent pupillary defect. In our case series, we find that the APD Tester ${ }^{\mathrm{TM}}$ can enhance diagnoses, and with its ease of use, may also be used by technicians and assistants for increased efficiency in clinic.
\end{abstract}

${ }^{*}$ Corresponding author: Timothy You, Orange County Retina Group, 1200 North Tustin Ave, Suite 140, Santa Ana, CA 92705, USA, Tel: +1 9497434317, E-mail: crystalhuangx@gmail.com

Citation: You TT, Huang CX, Chen S, Maggiano J, Rathod R, et al. (2017) The Reliability and Validity of a Handheld Relative Afferent Pupillary Tester in Diagnosing Optic Neuropathy. J Ophthalmic Clin Res 4: 032.

Received: May 14, 2017; Accepted: July 21, 2017; Published: August 04, 2017

\section{Introduction}

Pupillary light reflex is an important indicator of retina and optic nerve afferent input [1]. Asymmetric pupillary light reflex, also known as Marcus Gunn Pupil or Relative Afferent pupillary Defect (RAPD), is caused by lesions in the anterior visual pathway [2-4]. Its detection is useful for identifying retinal or optic nerve diseases $[1,5]$.

The standard technique for diagnosing RAPD is the alternating light test of Levatin, also known as the swinging flashlight test $[1,3,6$ 9]. While the patient looks at a distant target, the examiner shines a light alternately in each eye while observing pupillary response. Due to contraction anisocoria, which describes the greater pupillary response of the stimulated eye, RAPD diagnosis is only possible when one pupil's direct response is smaller than its consensual response [2]. Normal optic nerve function is indicated when the pupils react equally to direct and consensual light $[2,3]$. Concurrent pupillary constriction is followed by a small amount of dilation (hippus) each time the light is "swung" from one eye to the other [2,3]. In contrast, decrease of one pupil's initial constriction followed by dilation to a larger diameter, relative to the other pupil's response, indicates presence of an RAPD [2-4]. Loss of initial pupillary constriction on one side can also indicate presence of an RAPD [4].

Since the swinging flashlight test was first described in 1959, many modifications have been introduced [4]. These suggestions include magnification, effective for detection of subtle RAPD, with a slit-lamp biomicroscope or with high plus magnifying lens $[4,10]$. However, Lankaranian et al., found the use of the slit lamp to be inconvenient, while acknowledging the utility of the idea to improve detection with magnification [4]. Using only the swinging flashlight test, Lankaranian et al., suggested that almost half of the RAPD cases in a subject population will be missed [4]. Once RAPD has been detected, the detection may be quantified using such methods as neutral density filters [6,11], crossed polarized filters [11], the Sbisa bar [6], automated pupillometry [8] and pupillography [6]. The primary limitation with these methodologies and the swinging flashlight test is the examiner's inability to simultaneously view both eyes in evaluation of pupillary response.

We present a case series on the first reported use of the APD Tester $^{\text {max. }}$. This portable ophthalmic tool is the first reported device enabling examiners to observe both pupils simultaneously under high magnification, in a superimposed image [3]. We find that APD Tester ${ }^{\text {tw }}$ usage supports improved RAPD diagnosis and quantification.

\section{Materials and Methods}

The APD Tester ${ }^{\mathrm{rm}}$ (Richmond Products, Good-Lite Company) comprises high plus lenses, fixed LED lights and blue/UV lights, batteries and a sturdy casing, as shown in figure 1 [3]. The original APD Tester $^{\text {Tw }}(A P D+20)$ has a +20 diopter lens; while the newer model $(\mathrm{APD}+8)$ has less magnification with $\mathrm{a}+8$ diopter lens. Kamkar found that usage of $\mathrm{APD}+8$ was easier to learn and more comfortable for both examiners and patients. Currently, the APD Tester ${ }^{\text {rix }}(A P D+20$ and APD+8) costs $\$ 160$, while the second generation APD Tester ${ }^{\text {twx }}$ with variable light levels costs $\$ 300$. 


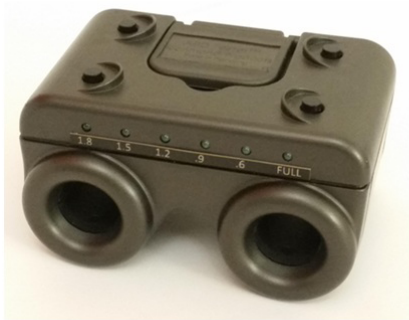

Figure 1a

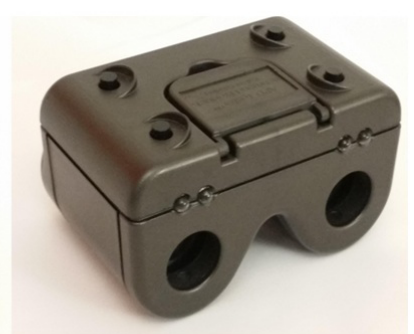

figure $1 \mathrm{~b}$
Figure 1: Photographs of the APD Tester ${ }^{\mathrm{TM}}$, from the examiner's point of view (Figure 1a) and the patient's point of view (Figure 1b).

With the second generation APD Tester ${ }^{\mathrm{nw}}$, each white LED light can be adjusted, in a controlled stepwise fashion, to be dimmed by $0.6,0.9,1.2,1.5$ and $1.8 \log$ units (The maximum luminance level was chosen for its efficacy and patient comfort, and each step of halving luminance corresponds to 0.3 "log units"). This capability is enabled by an internal circuit board, calibrated to match a neutral density filter, which ensures that each LED light produces the same level of light. The resultant dimming of each LED light is equivalent in effect to a standard neutral density filter held in front of a penlight while performing the swinging flashlight test.

RAPD can be quantified by the APD Tester ${ }^{\text {re }}$ with variable lights by the following method. Once RAPD has been detected in one eye, the white LED and UV buttons on the side of the normal eye can be held down for a few seconds, while the other white LED button is simultaneously pressed once. This will dim the LED light for the normal eye by $0.6 \log$ units, equivalent to a quarter of full luminance. If the examiner still detects RAPD, more light reduction is needed. The same process is repeated until the RAPD is neutralized, i.e., relative dilation is no longer observed. If the RAPD is reversed, the LED luminance must be increased. This is accomplished by holding down the same two LED and UV buttons, while pressing the other UV button. Once the test is done, the unit can be brought back to default full luminance by holding down all four buttons together for a few seconds.

Using APD+8, Kamkar and You examined 10 normal subjects and 10 abnormal subjects, with findings summarized in table 1 and table 2. Each patient was instructed to look at a distant object, while room lights were dimmed at a level allowing for visibility of the subject's pupils through the APD Tester ${ }^{\mathrm{Tw}}$. Initially, a diplopic view was seen, but slight focusing and convergence produced a single fused image. The superimposed, simultaneous view of both pupils enabled convenient comparison of pupillary sizes and responses. Direct responses were detected by shining the white LED light on each pupil using the corresponding top button; consensual responses were detected by shining the white LED light on one pupil, while observing the other pupil. Direct responses were easily compared, using the buttons to quickly switch white LED light illumination from one eye to the other, as shown in figure 2. A positive RAPD was present if repeated alternating of light between the two eyes elicited a relative momentary dilation of one pupil.

The grading of RAPD is on a scale of 1 to 5 , based on standards in the medical literature [12]. Bell and colleagues [12] used a 3 second pause time for each eye. Grade I indicated a weak initial constriction and greater redilatation; grade II, an initial stall and greater redilatation; grade III, an immediate pupillary dilatation; grade IV, an immediate pupillary dilatation following prolonged illumination of the

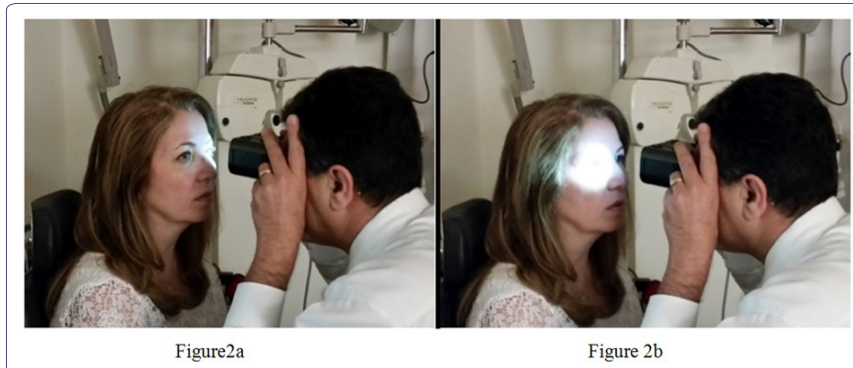

Figure 2: Photographs of APD Tester ${ }^{\mathrm{TM}}$ used by an examiner (right side of photographs) to sequentially examine the subject's left eye (Figure 2a) and right eye (Figure 2b).

good eye for 6 seconds; grade $\mathrm{V}$, immediate pupillary dilatation with no secondary constriction.

We limited the number of examiners to limit inter-operator variability. The study was a non-comparative test with the primary objective of determining device operational performance, i.e., to detect an RAPD if present in a patient with known optic neuropathy, and to document the absence of a pupillary defect in a normal patient.

\section{Results}

The APD Tester ${ }^{\text {Tw }}$ was used to examine the following 10 known normal subjects (Tables $1 \& 2$ ).

\section{Discussion}

Detection of RAPD is critical in assessing the optic nerve, which is subject to injury from different etiologies including trauma, compressive lesions, atrophy and inflammatory conditions [3]. RAPD detection may also play an important role in glaucoma patient assessment, because RAPD detection may precede visual field and optic disc damage [4]. Additionally, Tabatabaei et al., [5] report that higher grade of RAPD can predict final visual acuity in patients with traumatic optic neuropathy, Takayama et al., [8] find that RAPD outcomes are correlated rarely with visual acuity and AMD dimension and Folk et al., [13] find that pupillary defect is proportional to the extent of retinal detachment.

We find that the APD Tester ${ }^{\mathrm{Tm}}$ allows for convenient detection of RAPD, with many important applications and advantages over previous technologies. For instance, pupillography may provide important insight into the swinging flashlight test, but because of such reasons as lack of portability and technical limitations, it is not routinely performed during examinations [9]. Volpe and colleagues [9] note that a portable, affordable, consistent device to identify RAPDs would be useful in the care of patients with neurogenic vision loss. The APD Tester $^{\text {Tw }}$ directly addresses this need as a portable, affordable, easy-touse new technology, and it was used to test for RAPD in a variety of patients ranging from 20 to 82 years of age. We report that the APD Tester $^{\text {Tw }}$ overcomes the limitations of the swinging flashlight test, including unequal retinal illumination and end-point determination [4]. The second generation APD Tester ${ }^{\mathrm{Tw}}$ also offers an improved means of RAPD measurement, with its capability to reduce light luminance in the unaffected eye until no relative dilation is observed, without the obstruction of one pupil caused by traditional use of neutral density filters.

We also find that the APD Tester ${ }^{\text {Txt }}$ overcomes the difficulties that previous authors have noted in the examination of subjects with dark 
Citation: You TT, Huang CX, Chen S, Maggiano J, Rathod R, et al. (2017) The Reliability and Validity of a Handheld Relative Afferent Pupillary Tester in Diagnosing Optic Neuropathy. J Ophthalmic Clin Res 4: 032.

- Page 3 of $4 \cdot$

\begin{tabular}{|c|c|c|c|c|c|c|c|c|c|}
\hline Initials & Age & Sex & $\mathrm{CC}$ & Medical Hx & Medications & Ocular Dx & APD w/tester & BVA & Exam Date \\
\hline EB & 35 & $\mathrm{~F}$ & $\begin{array}{l}\text { Growth on } \\
\text { conjunctiva }\end{array}$ & Unremarkable & None & Pterygium, mild & Negative & OD $20 / 20$, OS $20 / 20$ & $3 / 7 / 2017$ \\
\hline LG & 20 & M & Blurry at far & Unremarkable & None & Myopia, mild & Negative & OD $20 / 20$, OS $20 / 20$ & $3 / 3 / 2017$ \\
\hline LA & 57 & $\mathrm{~F}$ & Blurry at near & Unremarkable & None & Presbyopia & Negative & OD $20 / 20$, OS $20 / 20$ & $3 / 2 / 2017$ \\
\hline HS & 45 & M & Blurry at near & Unremarkable & None & Presbyopia & Negative & OD $20 / 20$, OS $20 / 20$ & $3 / 1 / 2017$ \\
\hline SS & 69 & $\mathrm{~F}$ & Blurry at near & $\begin{array}{l}\text { Depression, acid } \\
\quad \text { reflux }\end{array}$ & Lexapro, Prilosec & $\begin{array}{l}\text { Compound hyperopic } \\
\text { astigmatism, presbyopia }\end{array}$ & Negative & OD $20 / 20$, OS $20 / 20$ & $3 / 1 / 2017$ \\
\hline RR & 61 & M & Blurry at near & $\begin{array}{l}\text { Hypertension, } \\
\text { depression }\end{array}$ & $\begin{array}{l}\text { Effexor, Remeron, } \\
\text { Vistaril, Zantac }\end{array}$ & Hyperopia, presbyopia & Negative & OD $20 / 20$, OS $20 / 20$ & $2 / 28 / 2017$ \\
\hline SB & 58 & M & Blurry at far & Hypertension & Amplodipine & High myopia & Negative & OD $20 / 20$, OS $20 / 20$ & $2 / 28 / 2017$ \\
\hline TB & 27 & $\mathrm{~F}$ & Blurry at far & Unremarkable & None & Myopia & Negative & OD $20 / 20$, OS $20 / 20$ & $2 / 27 / 2017$ \\
\hline IM & 30 & M & Blurry at far & Unremarkable & None & $\begin{array}{l}\text { Compound myopic } \\
\text { astigmatism }\end{array}$ & Negative & OD $20 / 20$, OS $20 / 20$ & $2 / 24 / 2017$ \\
\hline
\end{tabular}

Table 1: The APD Tester ${ }^{\mathrm{TM}}$ was used to examine the following 10 known normal subjects.

\begin{tabular}{|c|c|c|c|c|c|c|c|c|c|}
\hline Initials & Age & Sex & $\mathrm{CC}$ & Medical Hx & Medications & Ocular Dx & APD w/tester & BVA & Exam Date \\
\hline MS & 58 & M & Vision loss OD & Hypertension & None & NAION & $4+$ & $\begin{array}{l}\text { OD 20/300, } \\
\text { OS 20/25 }\end{array}$ & 2/7/2017 \\
\hline WC & 68 & M & Blurred vision OS & Unremarkable & None & NAION & $4+$ & $\begin{array}{c}\text { OD } 20 / 40, \text { OS } \\
20 / 150\end{array}$ & $3 / 7 / 2017$ \\
\hline BG & 82 & $\mathrm{~F}$ & Vision loss OS & Coronary artery disease, DM & Glyburide, Prednisone & Arteritic AION & $4+$ & $\begin{array}{c}\text { OD } 20 / 25 \text {, OS } \\
20 / 400\end{array}$ & $1 / 5 / 2017$ \\
\hline JK & 38 & $\mathrm{~F}$ & Vision loss OD & Multiple sclerosis suspect & None & Optic neuritis & $4+$ & $\begin{array}{l}\text { OD 20/100, } \\
\text { OS } 20 / 30\end{array}$ & $3 / 29 / 2017$ \\
\hline PM & 54 & $\mathrm{~F}$ & Blurred vision OU & Unremarkable & None & $\begin{array}{l}\text { Nutritional optic } \\
\text { neuropathy }\end{array}$ & $2+$ & $\begin{array}{c}\text { OD } 20 / 50, \text { OS } \\
20 / 80\end{array}$ & $3 / 8 / 2017$ \\
\hline DW & 64 & $\mathrm{~F}$ & Vision loss OD & Postvitrectomy & Alphagan-P & NAION & $3+$ & $\begin{array}{l}\text { OD } 20 / 300 \\
\text { OS } 20 / 40\end{array}$ & $12 / 14 / 2016$ \\
\hline MS & 59 & M & Visual field defect OD & Unremarkable & Aspirin & NAION & $4+$ & $\begin{array}{c}\text { OD 20/80, OS } \\
20 / 30\end{array}$ & $2 / 8 / 2017$ \\
\hline $\mathrm{BH}$ & 67 & $\mathrm{~F}$ & Vision loss OD & Unremarkable & Combigan, Lumigan & NAION, OAG & $4+$ & $\begin{array}{l}\text { OD 20/100, } \\
\text { OS } 20 / 50\end{array}$ & $4 / 13 / 2016$ \\
\hline CR & 29 & $\mathrm{~F}$ & Vision loss OS & Unremarkable & None & Optic neuritis & $3+$ & $\begin{array}{c}\text { OD } 20 / 15, \text { OS } \\
20 / 200\end{array}$ & $3 / 1 / 2017$ \\
\hline
\end{tabular}

Table 2: The APD Tester ${ }^{\mathrm{TM}}$ was used to examine the following 10 subjects, who had optic neuropathy in one eye.

irises, small pupils, or pupils that react slowly to direct or consensual light $[3,4,8]$. The ability to swiftly shine a light in either pupil while observing with a magnified view of both pupils enables convenient detection of RAPD in post-traumatic, post-surgical, or diseased eyes, with small pupils or dark irises [3].

\section{Conclusion}

The APD Tester ${ }^{\text {rix }}$ offers an improved means of assessment for optic nerve pathologies. With the swinging flashlight test's conventional methodology, examiners focus on just one pupil at a time. In contrast, the APD Tester ${ }^{\mathrm{Tw}}$ allows for simultaneous comparison of relative pupillary responses. Currently, two versions of the APD Tester ${ }^{\mathrm{Tn}}$ are available: the first generation model that functions as a screening device, and the second generation model that includes the function to grade or quantify RAPD for each eye in standard log units of a neutral density filter. The APD Tester ${ }^{\mathrm{rw}}$ thus has the potential to enhance diagnoses, and with its ease of use, it may also be used by technicians and assistants for increased efficiency in clinic.
For future studies, we recommend a comparison of RAPD quantification as measured by the traditional method with neutral density filters, with that as measured by the second generation APD Tester ${ }^{\text {Twx }}$ with variable LED lights, incorporating intra- and inter-observer variability.

\section{Acknowledgement}

Dr. Kamkar would like to acknowledge Dr. James Bailey of Marshall B. Ketchum University (SCCO), Lloyd Powell of Richmond Products, and Chris Greening of Good-Lite Company for believing in the APD Tester ${ }^{\mathrm{m}}$ and helping to bring it to the market.

\section{Conflicting Interest (If present, give more details)}

Dr. Kamkar has a relatively small financial interest, as a result of his intellectual property. He invented the APD Tester ${ }^{\mathrm{rm}}$.

\section{References}

1. Bergamin O, Zimmerman MB, Kardon RH (2003) Pupil light reflex in normal and diseased eyes: diagnosis of visual dysfunction using waveform partitioning. Ophthalmology 110: 106-114. 
2. Cox TA (1989) Pupillographic characteristics of simulated relative afferent pupillary defects. Invest Ophthalmol Vis Sci 30: 1127-1131.

3. Kamkar B (2015) Afferent pupil tester. US 9681805 B2 patent, USA.

4. Lankaranian D, Altangerel U, Spaeth GL, Leavitt JA, Steinmann WC (2005) The usefulness of a new method of testing for a relative afferent pupillary defect in patients with ocular hypertension and glaucoma. Trans Am Ophthalmol Soc 103: $200-208$

5. Tabatabaei SA, Soleimani M, Alizadeh M, Movasat M, Mansoori MR, et al. (2011) Predictive value of visual evoked potentials, relative afferent pupillary defect, and orbital fractures in patients with traumatic optic neuropathy. Clin Ophthalmol 5: 1021-1026.

6. McCormick A, Bhola R, Brown L, Squirrel D, Giles J, et al. (2002) Quantifying relative afferent pupillary defects using a Sbisa bar. Br J Ophthalmol 86 985-987.

7. Semmlow J, Hansmann D, Stark L (1975) Variation in pupillomotor responsiveness with mean pupil size. Vision Res 15: 85-90.
8. Takayama K, Ito Y, Kaneko H, Nagasaka Y, Tsunekawa T, et al. (2016) Cross-sectional pupillographic evaluation of relative afferent pupillary defect in age-related macular degeneration. Medicine (Baltimore) 95: 4978.

9. Volpe NJ, Plotkin ES, Maguire MG, Hariprasad R, Galetta SL (2000) Portable pupillography of the swinging flashlight test to detect afferent pupillary defects. Ophthalmology 107: 1913-1921.

10. Glazer-Hockstein C, Brucker AJ (2002) The detection of a relative afferent pupillary defect. Am J Ophthalmol 134: 142-143.

11. Rosenberg ML, Oliva A (1990) The use of crossed polarized filters in the measurement of the relative afferent pupillary defect. Am J Ophthalmol 110: $62-65$

12. Bell RA, Waggoner PM, Boyd WM, Akers RE, Yee CE (1993) Clinical grading of relative afferent pupillary defects. Arch Ophthalmol 111: 938-942.

13. Folk JC, Thompson HS, Farmer SG, O'Gorman TW, Dreyer RF (1987) Relative afferent pupillary defect in eyes with retinal detachment. Ophthalmic Surg 18: 757-759. 